\title{
A New Lattice Action For Studying Topological Charge.
}

\author{
Pilar Hernández and Raman Sundrum* \\ Lyman Laboratory of Physics, Harvard University, Cambridge, MA 02138, USA
}

\begin{abstract}
We review our recent proposal for a new lattice action for non-abelian gauge theories which reduces short-range lattice artifacts in the computation of the topological susceptibility. The standard Wilson action is replaced by the Wilson action of a gauge covariant interpolation of the original fields to a finer lattice. We illustrate the improved behavior of a same-philosophy new lattice action in the $O(3) \sigma$-model in two dimensions.
\end{abstract}

\section{INTRODUCTION}

Field configurations with non-zero topological charge are expected to have a strong influence on the dynamics of asymptotically free theories. The study of these effects however requires nonperturbative techniques and one would expect that ultimately Monte Carlo methods on the lattice would be best suited to it. The gold-plated observable is the topological susceptibility $\chi_{t}$, as inspired by the large- $N_{c}$ analyses. In the continuum it is given by,

$\chi_{t} \equiv \int d^{4} x<q(x) q(0)>\left.\right|_{\text {no quarks }}$,

$q(x)$ being the topological charge density,

$q(x)=\frac{1}{32 \pi^{2}} \epsilon_{\mu \nu \rho \sigma} \operatorname{Tr}\left[F_{\mu \nu} F_{\rho \sigma}\right]$.

The topological charge, $Q \equiv \int q(x)$, is an integer if the field strength vanishes at infinity or if (euclidean) space-time is compact. A continuum analysis shows that the action of any configuration with non-zero topological charge must satisfy the following bound,

$S \geq \frac{8 \pi^{2}|Q|}{g_{0}^{2}}$

which is saturated by instantons, $S^{\text {instanton }}=$ $8 \pi^{2} / g_{0}^{2}$. There are several choices for the operator $q(x)$ on the lattice. We will deal only with the geometrical definition due to Lüscher [1], which gives an integer-valued topological charge and does not require renormalization.

*Research supported by NSF-PHYS-92-18167.
The topological susceptibility in QCD is expected to scale as $\left(\mathrm{mass}^{4}\right.$ in the continuum limit. However, it was found [2] that the Wilson action gives rise to short-range fluctuations with nonzero geometrical topological charge and such a small action that they overwhelm the contribution of slowly varying fields and can destroy the expected scaling. It is easy to understand how these fluctuations called dislocations result from the mismatch between the geometrical definition of topological charge and the Wilson action. Consider a continuum instanton $A_{\mu}(y)$ which saturates the bound (3), and discretize it on a lattice of spacing $b$,

$U_{\mu}(s) \equiv P \exp \left(i \int_{s b}^{s b+b \hat{\mu}} d y A_{\mu}(y)\right)$,

where $s b$ are the sites of the $b$ lattice. The geometrical definition of topological charge assigns a non-zero value even to a lattice configuration (4) obtained from very small instantons, of $O(b)$. On the other hand, it is clear that the Wilson action approximates very poorly the continuum action for such rough configurations, and in fact it turns out to be smaller, strongly violating the bound (3). On the other hand, a semiclassical continuum analysis of dilute instantons indicates that for any theory in which the continuum susceptibility is well-defined, there exists an $\alpha<1$ such that, if the action of topologically non-trivial configurations is always larger than $\alpha S^{\text {instanton }}$, the susceptibility is ultraviolet finite [3]. Thus satisfying this minimum bound $S \geq \alpha S^{\text {instaton }}$ is a sufficient condition for scaling of the susceptibility. We will show that our new action satisfies this minimum bound. 


\section{NEW ACTION}

There are two important observations that led us to consider the new action proposed in [4]. The first is that the geometrical topological charge assigned to a lattice configuration is just the naive topological charge of a continuum configuration obtained by smoothly interpolating the lattice configuration. Then it is clear that if, instead of using the standard Wilson action of the original lattice configuration, we use the continuum action of the interpolated configuration, the continuum bound is necessarily satisfied, as first suggested in [5]. More concretely, in [6] we described a procedure to obtain a continuum gauge field $a_{\mu}(y)$ which interpolates any $b$-lattice configuration ${ }^{2}$. The interpolation is local and gauge covariant, i.e. for a $b$-lattice gauge transformation $\Omega(s)$,

$a_{\mu}\left[U^{\Omega}\right]=a_{\mu}^{\omega}[U]$,

where $\omega$ is a gauge transformation in the continuum. A geometrical topological charge of the $b$-lattice configuration is defined as the one associated to the interpolated field [6],

$Q=\frac{1}{32 \pi^{2}} \int d^{4} y \operatorname{Tr}\left[\tilde{f}_{\mu \nu}(y) f_{\mu \nu}(y)\right]$.

Now, it is clear that replacing the standard Wilson action by the continuum action of $a_{\mu}$, i.e.

$S^{\text {cont }}=\frac{1}{2 g_{0}^{2}} \int d^{4} y \operatorname{Tr}\left[f_{\mu \nu}(y) f_{\mu \nu}(y)\right]$,

ensures that the continuum bound is satisfied for the same reason as it is in the continuum. Notice that this is a perfectly gauge invariant action for the lattice field $U_{\mu}(s)$, by eq. (5). The only problem with the action (7) is that it is computationally impractical.

The second and central observation is that using (7) is in fact not necessary. An action defined on a finer lattice, with lattice spacing $f$ (we will take $b / f$ to be integer), that approximates $S^{c o n t}$ to just enough precision so that the minimum bound is satisfied, will ensure the scaling of the topological susceptibility, as explained above.

${ }^{2}$ The continuum field when discretized according to (4) gives back $U_{\mu}(s)$ ].
If the required ratio $f / b$ turns out to be not too small, the new action will be much easier to compute than (7).

More precisely, referring to $x$ as the sites on the $f$-lattice and $s$ as the sites on the $b$-lattice $\left(x_{\mu}=s_{\mu}+m_{\mu} \frac{f}{b}, m_{\mu}=0, \ldots, \frac{b}{f}-1\right)$, the interpolation procedure in [6] gives a set of link variables $u_{\mu}[U](x)$, such that

$-\frac{i}{f} \log \left(u_{\mu}(x)\right)=a_{\mu}(x)+O\left(f / b^{2}\right)$,

where $a_{\mu}(x)$ is the continuum interpolation discussed above, at point $x$. On the $f$ lattice, we can simply choose the standard Wilson action. The partition functional will then have the form,

$\mathcal{Z}=\int \prod_{s} \mathcal{D} U(s) e^{-S_{\text {wilson }}^{f}[u[U]]}$,

where, $D U$ is the usual Haar measure for nonabelian gauge fields on the $b$-lattice, and the Wilson action in terms of the interpolated link variables $u[U]$ is given by,

$S_{\text {wilson }}^{f}[u[U]]=\frac{1}{g_{0}^{2}} \sum_{x} \sum_{\mu \neq \nu}\left(I-u_{\mu \nu}[x]+\right.$ h.c. $)$,

with $u_{\mu \nu}[x]$ being the $f$-plaquette variable. Again, this action is gauge invariant, because the functional $u[U]$ is gauge covariant [6] and from eq. (8) it then follows that,

$S_{\text {wilson }}^{f}=\frac{1}{2 g_{0}^{2}} \int d^{4} y \operatorname{Tr}\left[f_{\mu \nu}(y) f_{\mu \nu}(y)\right]+O(f / b)$.

Determining how small the ratio $f / b$ must be to satisfy $S \geq \alpha S^{\text {instanton }}$ requires a numerical analysis. In the next section we present a first numerical study of this issue in a simplified model in two dimensions. The results encourage us to believe that $\frac{f}{b}$ need not be very small in order to recover scaling of $\chi_{t}$.

\section{O(3) $\sigma$-MODEL IN 2D}

As is well known the $\mathrm{O}(3) \sigma$-model in two dimensions shares many similarities with YangMills [7]. In the continuum, the model is defined by the action,

$S=\frac{1}{2 g_{0}^{2}} \int d^{2} x \sum_{\mu}\left(\partial_{\mu} \vec{n}(x)\right)^{2}$, 
where $\vec{n}$ is a 3 -component real field satisfying the constraint $\vec{n}^{2}=1$. The continuum topological charge in this model measures the number of times that space-time wraps around the $\vec{n}$-sphere. In a standard lattice treatment the action, $S^{b}$, is the naive discretization of (11), while the geometrical topological charge $Q^{b}$ was first defined on the lattice in [3]. In the continuum, all topologically non-trivial configurations satisfy $S \geq 4 \pi|Q| / g_{0}^{2}$, however, the standard action $S_{b}$ gives rise to dislocations, i.e. configurations with $S^{b}<4 \pi\left|Q^{b}\right| / g_{0}^{2}$ 3]. These artifacts disappear when the action is improved along the lines of the previous section. The new action, $S^{f}$, is defined on a finer lattice, where the spin variables are obtained from a geodesic interpolation of the original variables (see 4 for explicit formulae). This new action then satisfies $S^{f} \geq \frac{4 \pi\left|Q^{b}\right|}{g_{0}^{2}}+O(f / b)$ 《t.

As a first numerical study of the improvement as a function of the ratio $f / b$, we considered the discretization of continuum instanton configurations with unit topological charge [7] (which saturate the continuum bound). Such configurations are characterized by a radius $r$ and a center that we fix at the center of the volume to reduce finite volume effects. Figure 1 summarizes our results. It represents the action of the discretized instanton configuration as the radius is varied, normalized to the continuum one-instanton bound. Generically there is always a critical, $r_{c} \sim O(b)$, below which $Q^{b}=0$ (vertical line). The continuous curve corresponds to the standard action, while the dashed ones correspond to the improved action for different values of the ratio $f / b$. It is clear that the standard action is problematic, since for $r>r_{c}$ the action is considerably smaller than the continuum bound. For the new action however the continuum bound is satisfied already for a ratio of $f / b$ as large as $1 / 2$ ! Furthermore, as the instanton is shrunk to sizes of $O(b)$, a small barrier develops, separating the $Q^{b}=0$ and $Q^{b}=1$ sectors. This is expected because the interpolation of a discretized instanton of size $O(b)$ is generically no longer an instanton (and it is further from it as the radius of the original instanton decreases). This is why the action of configurations with $r>r_{c}$ increases as $r \rightarrow r_{c}$

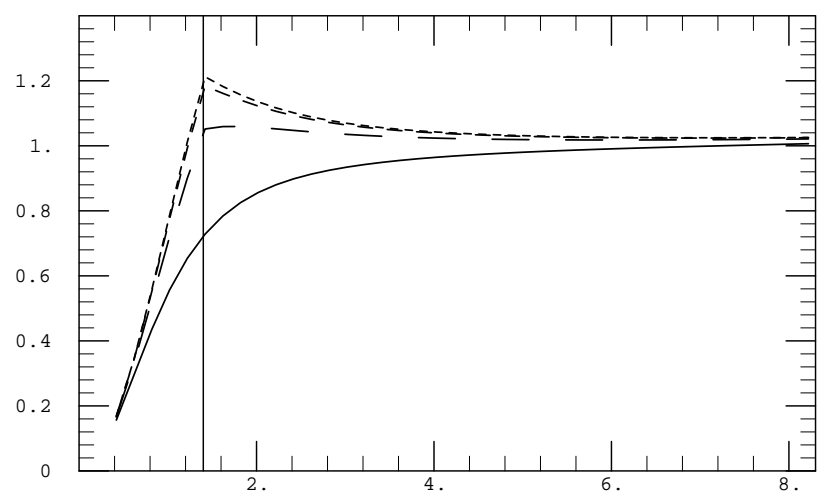

Figure 1. Action of a discretized instanton of the $\mathrm{O}(3)$ model (normalized to $4 \pi / g_{0}^{2}$ ) as a function of its radius, $r$. The full line is the standard action in a $100 \times 100$ lattice and the dashed lines correspond respectively to $f / b=1 / 2,1 / 4,1 / 6$ (smaller $f / b$, smaller dashing). The vertical line at $r_{c} \sim 1.4 b$ separates the $Q^{b}=0,1$ sectors.

near $r_{c}$.

Although the results for the $O(3)$ model are very promising, a numerical analysis is needed in the Yang-Mills case to determine the required ratio $f / b$ there. If it turns out to be moderate, the action (10) should be practical in MC simulations.

\section{REFERENCES}

1. M.Lüscher, Comm.Math.Phys.85(1982)39.

2. D.J.R.Pugh, M.Teper, Phys. Lett. 224B (1989) 159. M. Göckeler et al., Phys. Lett. 233B (1989) 192 and references therein.

3. B.Berg, M.Lüscher, Nucl. Phys. B190 (1981) 412. M.Lüscher, Nucl. Phys. B200(1982)61.

4. P.Hernández, R.Sundrum, hep-lat/9604009.

5. M.Göckeler et al., Nucl.Phys.B404(1993) 839.

6. P. Hernández, R. Sundrum, Nucl. Phys. B472(1996) 334 and these proceedings.

7. E.g. Gauge Fields and Strings, A.M. Polyakov. 